\title{
DECISÕES AUTOMATIZADAS E O DIREITO À EXPLICAÇÃO NO REGULAMENTO GERAL DE PROTEÇÃO DE DADOS DA UNIÃO EUROPÉIA
}

\author{
AUTOMATED DECISIONS AND THE RIGHT TO EXPLAIN IN THE \\ GENERAL DATA REGULATION OF THE EUROPEAN UNION
}

\author{
Leticia Canut
}

\section{RESUMO}

No contexto de preocupações advindas da sociedade e economia de dados, marcadas por software e algoritmos de Inteligência Artificial, responsáveis por decisões automatizadas no âmbito de diferentes atividades humanas, o presente artigo parte da problemática de saber se e em que medida o Regulamento Geral de Proteção de Dados da União Européia-GDPR criou um direito à explicação das decisões automatizadas. Intenta-se analisar o quadro de discussões acerca desse direito no contexto do GDPR para verificar a hipótese de que a partir de diversos dispositivos do GDPR teria sido previsto tal direito à explicação. 0 objetivo do trabalho consiste na apresentação e análise do quadro de debates acerca da existência do direito à explicação a partir do GDPR. Os objetivos específicos, identificados nos tópicos do trabalho, consistem em o apresentar as decisões automatizadas no contexto da sociedade e economia de dados; apresentar previsões mais relevantes do GDPR acerca das decisões automatizadas; analisar posicionamentos acerca da previsão do direito á explicação das decisões automatizadas a partir do GDPR. Para tanto, recorre-se á pesquisa descritiva, qualitativa e exploratória, a partir do método dedutivo, com recurso aos procedimentos de pesquisa documental, bibliográfico e pesquisa eletrônica.

Palavras-chave : Direito à explicação. Decisões automatizadas. Regulamento Geral de Proteção de Dados. 


\begin{abstract}
In the context of concerns from data society and data economy, tagged by software and Artificial Intelligence algorithms that are responsible for automated decisions within the scope of different human activities, this article aims to solve the problem of whether and to what extent the European Union's General Data Protection Regulation-GDPR has created a right to explain automated decisions. This research analyses the discussion framework on this right in the context of the GDPR to verify the hypothesis that from different devices of the GDPR this right to explanation would have been provided. The work aims to present and examine the framework of debates on the existence of the right of explanation from the GDPR. The specific objectives, identified in the article topics, are: to present automated decisions in the context of data society and data economy; present more relevant GDPR predictions about automated decisions; analyze positions regarding the prediction of the right to explain automated decisions from the GDPR. For this purpose this work will be elaborated from descriptive, qualitative and exploratory research, using the deductive method, using documentary, bibliographic and electronic research procedures.
\end{abstract}

Keywords: Right to explanation. Automated decisions. General Data Protection Regulation.

\title{
INTRODUÇÃo
}

Na sociedade e economia de dados, os software e algoritmos se tornaram elementos centrais, desempenhando, dia-a-dia, papéis cada vez mais relevantes na vida das pessoas em variados ramos de atividades, o que tem chamado a atenção de pesquisadores de diversas áreas do conhecimento.

O destaque das potencialidades positivas de sistemas baseados em algoritmos de diferentes tipos de Inteligência Artificial-IA não é novidade. No entanto, com o desenvolvimento das Tecnologias de Informação e Comunicação-TICs, especialmente no âmbito do que vem sendo chamado de Big Data, o recurso a algoritmos de IA para a tomada de decisões automatizadas tem despertado interesse, também, em razão das potencialidades negativas que estão a gerar.

Dentre as potencialidades negativas mais estudadas estão: discriminação que podem gerar em razão de questões de gênero, racial, 
localidade, poder aquisitivo, etnia, dentre outros fatores que nos sistemas de IA podem ser analisados e combinados de forma rápida e sem muitos esforços; a sua obscuridade ou falta de transparência que, de um lado, inviabiliza que as pessoas saibam que tais decisões estejam sendo tomadas por sistemas inteligentes ou, ainda que saibam da sua existência, não conseguem compreender como tal decisão foi alcançada.

Ao realizar pesquisas em busca de alternativas para lidar com as potencialidades negativas das decisões automatizadas, a pesquisadora deparou-se com artigos e notícias sobre "o direito á explicação" ou "direito de explicação" das decisões automatizadas. 0 tema soou inovador e extremamente relevante já que a pesquisa e compreensão desse "novo direito" poderia contribuir para os debates sobre a obscuridade e discriminação decorrentes das decisões algorítmicas. Diante desse cenário resolveu-se enfrentar a temática.

Num primeiro momento, buscou-se identificar qual era o fundamento e contexto no qual se estava a discutir esse direito tão inovador. Foi então que, por meio de pesquisas em artigos nacionais e, especialmente, internacionais, identificou-se que o debate estava sendo construído a partir das previsões da GDPR, antes mesmo de sua entrada em vigor, que se deu em maio de 2018. Percebeu-se que artigos de $2017^{1}$ marcaram o início dos debates sobre a temática e tiveram importantes repercussões em publicações posteriores.

Da leitura inicial, necessária para elaboração do projeto de pesquisa, pretendia-se delimitar o tema para analisar o direito de explicação das decisões automatizadas a partir das previsões do GDPR. Já na elaboração do projeto e das leituras preliminares tal delimitação demonstrou-se inviável já que se encontrou um campo repleto de posicionamentos divergentes acerca da própria existência desse direito.

A partir disso, e enfrentando tal temática pela primeira vez, optou-se por uma nova delimitação do tema por meio da qual foi colocado como objeto de análise as discussões acerca do direito à explicação das decisões automatizadas no contexto do Regulamento Geral de Proteção de Dados da União Européia - GDPR².

Tendo em vista o atual modelo de sociedade e de economia, dirigidos intensamente por dados e por tecnologias voltadas ao tratamento de tais 
dados, com utilização de algoritmos complexos, baseados em sistemas de aprendizado de máquina e em inteligência artificial, partiu-se do problema de saber se e em que medida o GDPR criou ou determinou um novo direito sob a denominação de direito à explicação ou direito de explicação das decisões automatizadas.

A hipótese principal, e inicial, apresentada para o projeto da pesquisa foi de que a partir de diversos dispositivos do GDPR teria sido previsto tal direito à explicação. Para verificar a hipótese apresentada foi elaborada pesquisa descritiva, qualitativa e exploratória, feita a partir do método dedutivo, com recurso aos procedimentos de pesquisa documental, com destaque para o GDPR, e bibliográfico, com foco, especialmente, em artigos científicos estrangeiros. Para ambos os procedimentos de pesquisa socorreu-se á pesquisa eletrônica.

Estabeleceu-se como objetivo do trabalho a apresentação do quadro de debates ${ }^{3}$ acerca da existência do direito à explicação a partir do GDPR. Buscou-se traçar um panorama inicial que fosse capaz de contribuir, especialmente no âmbito nacional ${ }^{4}$, para a discussão de tema tão caro e tão essencial para a proteção de diversos direitos fundamentais dos cidadãos na sociedade e economia de dados.

Para alcançar esse objetivo o trabalho foi dividido em três tópicos, além da introdução e considerações finais: o primeiro para apresentar, brevemente, o espaço ocupado pelas decisões automatizadas na sociedade e economia atuais, baseadas em dados; o segundo para apresentar algumas das previsões mais relevantes do GDPR acerca das decisões automatizadas; o terceiro para analisar alguns posicionamentos acerca da previsão do direito á explicação das decisões automatizadas a partir do GDPR.

\section{DECISÕES AUTOMATIZADAS NA SOCIEDADE E ECONOMIA DE DADOS}

O sociólogo Manuel Castells refere-se à formação de um novo paradigma na década de 70 do século XX, o da tecnologia da informação ${ }^{5}$, e à consequente emergência de uma sociedade e economia informacionais 
(CASTELLS, 1999, p.40-209), denominadas por alguns de digitais dentre tantas outras denominações atribuídas (CANUT, 2007, p.47-52).

Para Castells (1999, p.55;69), passou-se de um modo de produção industrial para o informacional, no qual "as tecnologias de geração de conhecimentos, de processamento da informação e de comunicação de símbolos" tornaram-se" a fonte de produtividade". Trata-se de um contexto em que o elemento central encontra-se na aplicação de conhecimentos e de informação "para a geração de conhecimentos e de dispositivos de processamento/comunicação da informação, em um ciclo de realimentação cumulativo entre inovação e seu uso" e não na informação, em si, e no conhecimento.

É nesse sentido que a nova economia "é informacional porque a produtividade e a competitividade de unidades ou agentes nessa economia (sejam empresas, regiões ou nações) dependem basicamente de sua capacidade de gerar, processar e aplicar de forma eficiente a informação baseada em conhecimentos"(CASTELLS, 1999, p.119).

Com o avanço das Tecnologias de Informação e Comunicação ao longo dos anos e das novas potencialidades por elas criadas, observa-se que as tecnologias de processamento, geração e tratamento passam a atuar não apenas sobre a informação, mas sobre quaisquer dados ${ }^{6}$, estejam eles estruturados ou não estruturados.

Nesse contexto de criação, coleta, armazenamento, tratamento, disseminação e transferência exponenciais de dados, de aumento da capacidade de seu processamento e do barateamento das tecnologias voltadas para esse fim, passa-se a falar em sociedade e economia de dados(EUROPEAN COMMISSION,2017; GRELLER apud PUGLIESI, BRANDÃO).

Num cenário repleto de espaços privilegiados para coleta de dados - como, por exemplo, as redes sociais, a Internet das Coisas, e a própria aceleração desses processos pelos smartphones (PUGLIESI, BRANDÃO, 2015, p.455) torna-se possível traçar perfis completos que permitem identificar preferências pessoais, hábitos, grupos de consumidores específicos e marketing personalizado ou novas estratégias de marketing (TOMASEVICIUS FILHO, 2015, p.52; NOVO, AZEVEDO, 2014, p.6), 
a influência no comportamento dos usuários, podendo, ainda, gerar exclusões e discriminações em diversos campos sociais.

O Regulamento Geral de Proteção de Dados da União Européia, conhecido pela sigla GDPR, conceitua o tratamento de dados pessoais como

[...] uma operação ou um conjunto de operações efetuadas sobre dados pessoais ou sobre conjuntos de dados pessoais, por meios automatizados ou não automatizados, tais como a recolha, o registro, a organização, a estruturação, a conservação, a adaptação ou alteração, a recuperação, a consulta, a utilização, a divulgação por transmissão, difusão ou qualquer outra forma de disponibilização, a comparação ou interconexão, a limitação, o apagamento ou a destruição (JORNAL OFICIAL DA UNIÃO EUROPÉIA).

Para tratar o enorme conjunto de dados que se forma, estruturados e não estruturados -chamados de Big Data (ARTHUR,2013)- recorre-se à inovação baseada em dados que envolve serviços de armazenagem de dados, de rede, uso de programas e de computadores, os software e seus algoritmos, um cenário em que se desenvolvem questões como a computação na nuvem- cloud computing- e mineração de dados-data mining (OECD, 2015; BRASIL, 2016). Temas que se tornam essenciais quando se tem em vista a proteção dos dados pessoais e da privacidade.

As tecnologias de Big Data concentram-se, principalmente, em "encontrar correlações - reconhecer padrões [...]" por meio da utilização de algoritmos de indução que permitem a "navegação no oceano de dados que se está imerso, possibilitando a extração de informações úteis, [...]”( PUGLIESE, BRANDÃO, 2015, p.461;462;463).

0 algoritmo pode ser compreendido como "a parte técnica essencial do software que diz respeito ao processo escolhido para solução de um problema determinado, ou seja, é como um programa de computador irá realizar determinada tarefa". Desta forma, a sequência de etapas para instruir a máquina a resolver um problema ou mesmo um conjunto de problemas é determinada por algoritmos ${ }^{7}$ (CANUT, MEDEIROS, 2017, p. 1042).

Na sociedade e economia de dados há uma grande variedade de algoritmos para lidar com o tratamento de dados pessoais.Eles 
desempenham diferentes funções e apresentam, também, diferentes níveis de complexidade.

Michael S. Gal e Niva Elkin-Korren (2017,p.6) explicam que há algoritmos: de nível muito básico para fornecer informações de acordo com escolhas do usuário, outros que coletam e organizam informações relevantes que são apresentadas pelos fornecedores, enquanto outros se dedicam a fornecer informações sobre qualidade de produtos e serviços; outros mais sofisticados que, com base em dados coletados, fazem previsões e/ou restringem opções apresentando somente aquelas que são consideradas relevantes com base nas características do usuário assim como em suas preferências reveladas no passado; e outros ainda mais sofisticados que fazem sugestões de produtos e serviços que possam ser consumidos conforme a capacidade financeira e no perfil de consumo do usuário. Esses algoritmos ajudam o usuário a tomar decisões(GAL, ELKIN- KORREN,2017, p.6,7).

Além disso, fala-se em uma nova geração de algoritmos que fazem e executam decisões comunicando-se diretamente com outros sistemas por meio da rede (GAL, ELKIN- KORREN,2017, p.6,7).

Algoritmos têm sido usados há muito tempo para auxiliar a tomada de decisões, mas nos últimos anos o crescimento do 'big data' e do 'aprendizado de máquina' tem impulsionado um aumento nas tomada de decisão algorítmicas nos mais variados setores (SCIENCE ANDA TECHNOLOGY COMMITTEE, 2018, p.3). De acordo com a A Royal Society

[...] o aprendizado de máquina oferece a possibilidade de estender processos automatizados de tomada de decisão, permitindo maior alcance e profundidade de tomada de decisão sem a intervenção humana", os usos potenciais são vastos e continua a crescer 'a uma taxa sem precedentes' (apud SCIENCE ANDA TECHNOLOGY COMMITTEE, 2018, p.9).

Nota-se que "os aplicativos de aprendizado de máquina estão testemunhando taxas de adoção sem precedentes devido à sua capacidade de melhorar radicalmente a tomada de decisões baseada em dados a um custo e escala incomparáveis para os seres humanos", tanto no setor privado quanto no setor público ${ }^{8}$ (VOGL, FARHANGI, CASEY, 2018). Assim, 
algoritmos inteligentes fazem parte do dia-a-dia das pessoas e elas nem percebem.(TAURION, 2016).

Um cenário marcado pela Inteligência artificial em que, cada vez mais, os algoritmos de aprendizado de máquina, responsáveis pelo processamento de grandes quantidades de dados, exercerão papel maior na regulação da vida das pessoas (VOGL, FARHANGI, CASEY, 2018), o que tem levado a um aumento das decisões automatizadas.

Nesse cenário, são levantadas diversas questões éticas acerca dos algoritmos como, por exemplo: sua opacidade(BURREL,2016; VIETH, BRONOWICKA,2015) e complexidade que afetam sua transparência e, consequentemente, não viabilizam o conhecimento dos princípios que estão por trás da função algorítmica já que podem consistir em segredo comercial ou objeto de proteção de propriedade intelectual, o que inviabiliza ao sujeito avaliar o comportamento do algoritmo; sua capacidade de manter as informações longe das pessoas, decidindo o que recebe atenção e o que deve ser ignorado; podem gerar preconceitos; tomam decisões subjetivas sem que se saiba como eles funcionam, sendo que às vezes nem mesmo os programadores podem prever como um determinado algoritmo irá decidir sobre um determinado caso (VIETH, BRONOWICKA, 2015)

A opacidade dos algoritmos pode ser de diferentes tipos e níveis, a depender do contexto de sua criação/aplicação e ao tipo de sujeito envolvido na relação, o que resulta em cenários diferenciados a serem enfrentados pelos operadores do direito.

Importante ressaltar que nesse contexto de obscuridade um dos aspectos que tem sido mais debatido consiste nos preconceitos ou tratamentos diferenciados decorrentes das decisões algorítmicas.

O conhecimento sobre as interferências dos algoritmos na tomada de decisões ou mesmo nas decisões automatizadas ainda é muito limitado e o tema tem sido objeto de estudos e políticas públicas internacionais e, também, nacionais. Apesar de tais iniciativas, trata-se de realidade que tem gerado crescente sentimento de impotência e perda de controle em relação ao tratamento de dados pessoais e à tutela de diferentes direitos fundamentais. 


\section{AS DECISÕES AUTOMATIZADAS NO REGULAMENTO GERAL DE PROTEÇÃO DE DADOS DA UNIÃO EUROPÉIA ${ }^{9}$}

Reconhecendo que as novas tecnologias transformaram a vida social e a economia, e tendo em vista os desafios que a evolução tecnológica apresentou e vem apresentando para a proteção de dados pessoais, especialmente diante do aumento significativo de sua coleta, compartilhamento e utilização, sem precedentes, por empresas privadas e por entidades públicas, em suas atividades, a União Européia, preocupada em garantir tanto a livre circulação de dados pessoais assim como um alto nível de proteção de dados (JORNAL OFICIAL DA UNIÃO EUROPÉIA, 2016, p.2) editou o General Data Protection Regulation-GDPR ( Regulamento Geral de Proteção de Dados) que entrou em vigor em maio de 2018.

Nesse sentido, o artigo $1^{\text {o }}$ (1) do regulamento "estabelece as regras relativas à proteção das pessoas singulares no que diz respeito ao tratamento de dados pessoais e à livre circulação desses dados". Sendo o direito à proteção dos dados pessoais considerado um direito fundamental (considerando 1 e art. $1^{\text {o }}$ (2)).

0 regulamento foi elaborado com intuito de gerar confiança viabilizadora do desenvolvimento da economia digital no mercado interno da União Européia por meio da previsão de um quadro normativo que se ocupa em fornecer "segurança jurídica e a segurança prática para as pessoas singulares, os operadores económicos e as autoridades públicas" (JORNAL OFICIAL DA UNIÃO EUROPÉIA, 2016, p.2).

O objeto de proteção são dados das pessoas singulares no contexto em que haja qualquer tipo de ligação com uma atividade comercial ou empresarial, estando excluídos de seu âmbito de aplicação "o tratamento de dados pessoais efetuado por pessoas singulares no exercício de atividades exclusivamente pessoais ou domésticas[...]" (JORNAL OFICIAL DA UNIÃO EUROPÉIA, p. 3,4). Além disso, o regulamento será aplicado para a proteção dos dados pessoais que recebam tratamento - no sentido amplo do conceito apresentado no tópico anterior, conforme o artigo 4o (2) do regulamento - tanto manual quanto automatizados (JORNAL OFICIAL DA UNIÃO EUROPÉIA, 2016, p.3). 
Sob esse aspecto, ao dispor sobre o âmbito de aplicação material do regulamento, o artigo $2^{\circ}$ prevê que o (1) [...] regulamento aplicase ao tratamento de dados pessoais por meios total ou parcialmente automatizados, bem como ao tratamento por meios não automatizados de dados pessoais contidos em ficheiros ou a eles destinados."

A normativa conceitua Dados pessoais como a [...] informação relativa a uma pessoa singular identificada ou identificável ${ }^{10}$ («titular dos dados») [...] "(art. 4ํㅜ (1). Outro conceito importante de ser apresentado para os fins pretendidos no presente trabalho, dentre todas conceituações feitas pelo Regulamento, consiste na Definição de perfis como

[...] qualquer forma de tratamento automatizado de dados pessoais que consista em utilizar esses dados pessoais para avaliar certos aspetos pessoais de uma pessoa singular, nomeadamente para analisar ou prever aspectos relacionados com o seu desempenho profissional, a sua situação económica, saúde, preferências pessoais, interesses, fiabilidade, comportamento, localização ou deslocações ${ }^{11}$ ( $\operatorname{art.4}{ }^{\circ}$ (4) ).

Apesar dessa breve apresentação, o presente tópico não pretende apresentar o GDPR, seu campo de atuação, se ocupar com todos os seus princípios - para os quais foi dedicado o capítulo II da normativa- os direitos por ele garantidos, a imposição de restrições possíveis, as infrações e sanções previstas, formação de autoridades competentes para sua aplicação, dentre tantos outros pontos relevantes.

Ocupa-se, tão somente, em abordar, de forma geral e resumida, os dispositivos mais relevantes (e mais citados), obrigatórios e não obrigatórios, e princípios relacionados à proteção de dados pessoais no contexto de decisões automatizadas. Apesar da importância dos demais princípios, direitos e dispositivos do regulamento e da sua interrelação com aqueles a serem aqui analisados, a delimitação é feita para que seja possível, no espaço reservado a um artigo científico, alcançar o objetivo proposto.

Dentre os princípios relativos ao tratamento de dados pessoais apresentados no artigo 5oㅡ, encontra-se o da transparência que além de ser apresentado, também, nos considerados do regulamento, foi previsto no capítulo que dispõe sobre os direitos do titular dos dados, no artigo 
12 da seção 1, sobre "transparência das informações, das comunicações e das regras para exercício dos direitos dos titulares dos dados".

"O princípio da transparência exige que qualquer informação destinada ao público ou ao titular dos dados seja concisa, de fácil acesso e compreensão, bem como formulada numa linguagem clara e simples, e que se recorra, adicionalmente, à visualização sempre que for adequado"(JORNAL OFICIAL DA UNIÃO EUROPÉIA, 2016, p.7;10).

Além disso, é exigência dos princípios do tratamento equitativo e transparente que

[...] o titular dos dados seja informado da operação de tratamento de dados e das suas finalidades. [...] 0 titular dos dados deverá também ser informado da definição de perfis e das consequências que daí advém. Sempre que os dados pessoais forem recolhidos junto do titular dos dados, este deverá ser também informado da eventual obrigatoriedade de fornecer os dados pessoais e das consequências de não os facultar.[...] (JORNAL OFICIAL DA UNIÃO EUROPÉIA, 2016, p.12).

O considerando 63 do GDPR refere-se ao direito do titular de dados de conhecer e de ser informado sobre diversas questões, dentre as quais encontra-se a "[...]lógica subjacente ao eventual tratamento automático dos dados pessoais e, pelo menos quando tiver por base a definição de perfis, das suas consequências.[...]" (JORNAL OFICIAL DA UNIÃO EUROPÉIA, 2016, p.12).

De acordo com o considerando 71,

O titular dos dados deverá ter o direito de não ficar sujeito a uma decisão, que poderá incluir uma medida, que avalie aspectos pessoais que lhe digam respeito, que se baseie exclusivamente no tratamento automatizado e que produza efeitos jurídicos que lhe digam respeito ou o afetem significativamente de modo similar, como a recusa automática de um pedido de crédito por via eletrónica ou práticas de recrutamento eletrónico sem qualquer intervenção humana. Esse tratamento inclui a definição de perfis mediante qualquer forma de tratamento automatizado de dados pessoais para avaliar aspectos pessoais relativos a uma pessoa singular, em especial a análise e previsão de aspectos relacionados com o desempenho profissional, a situação económica, saúde, preferências ou interesses pessoais, fiabilidade ou comportamento, localização ou deslocações do titular dos dados, quando produza efeitos jurídicos que lhe digam respeito ou a afetem 
significativamente de forma similar. No entanto, a tomada de decisões com base nesse tratamento, incluindo a definição de perfis, deverá ser permitida se expressamente autorizada pelo direito da União ou dos Estados-Membros aplicável ao responsável pelo tratamento, [...] Em qualquer dos casos, tal tratamento deverá ser acompanhado das garantias adequadas, que deverão incluir a informação específica ao titular dos dados e o direito de obter a intervenção humana, de manifestar o seu ponto de vista, de obter uma explicação sobre a decisão tomada na sequência dessa avaliação e de contestar a decisão. Essa medida não deverá dizer respeito a uma criança. JORNAL OFICIAL DA UNIÃO EUROPÉIA, 2016, p.14)

Esse mesmo considerando, tendo em vista a transparência e o tratamento equitativo, estabelece que o responsável pelo tratamento de dados deverá, dentre outras estipulações, utilizar procedimentos estatísticos e matemáticos adequados à definição de perfis, proteger os dados pessoais tendo em vista os riscos para os titulares do direito de dados e de forma a previr, "por exemplo,"[...] efeitos discriminatórios contra pessoas singulares em razão da sua origem racial ou étnica, opinião política, religião ou convicções, filiação sindical, estado genético ou de saúde ou orientação sexual, ou a impedir que as medidas venham a ter tais efeitos". Nesse sentido, dispõe que a utilização de categorias especiais de dados pessoais ${ }^{12}$ só deverá ser permitida em decisões automatizadas e na definição de perfis, em condições específicas (JORNAL OFICIAL DA UNIÃO EUROPÉIA,2016, p.15).

Além das menções feitas até aqui, há ao menos mais quatro artigos do regulamento que são referência para o debate acerca das decisões automatizadas, alguns a serem observados em sua totalidade e outros parcialmente.

0 artigo 13 prevê o direito dos titulares de dados pessoais obterem informações quando os dados forem coletados junto ao titular. Para além das informações constantes no artigo 13 (1), o artigo 13 (2), para garantir um processamento justo e transparente, estabelece informações adicionais a serem fornecidas, dentre as quais, encontra-se a de que a "existência de decisões automatizadas, incluindo a definição de perfis, referida no artigo 22 (1) e (4), e, pelo menos nesses casos, informações significativas sobre a lógica subjacente, bem como a importância e as consequências previstas de tal tratamento para o titular dos dados (f)" . 
Para as situações em que os dados pessoais não foram obtidos junto ao titular, o artigo 14 estabelece que para além das informações do artigo 14 (1), também deverão ser fornecidas pelo responsável pelo tratamento de dados, a fim de garantir um tratamento transparente e equitativo, aquelas relativas à "( $\mathrm{g}$ ) existência de decisões automatizadas, incluindo a definição de perfis referida no artigo 22 (1) e (4) e, pelo menos nesses casos, informações significativas sobre a lógica subjacente, bem como a importância e as consequências previstas de tal tratamento para o titular dos dados".

Ao tratar do direito de acesso do titular aos dados, o artigo 15 (1) estabelece que "o titular dos dados tem o direito de obter do responsável pelo tratamento a confirmação de que os dados pessoais que lhe digam respeito são ou não objeto de tratamento e, se for esse o caso, o direito de ter acesso aos seus dados pessoais" e outras informações, dentre as quais: (h) a existência de decisões automatizadas, incluindo a definição de perfis, referida no artigo 22 (1) e (4), e, pelo menos nesses casos, informações significativas sobre a lógica subjacente, bem como a importância e as consequências previstas de tal tratamento para o titular dos dados.

Em algumas abordagens sobre o tema tratado nesse tópico observase a citação do artigo 21 do regulamento que, assim como o artigo 22, a ser citado na sequência, consta da seção 4, do Capítulo III -Direitos do titular dos dados- que trata do direito de oposição e decisões individuais automatizadas. 0 artigo 21(1) prevê que "o titular dos dados tem o direito de se opor, por motivos relacionados com a sua situação particular, a qualquer momento ao tratamento de dados pessoais que lhe digam respeito, com base nas alíneas e) ou f) do artigo 6으 (1), incluindo perfis baseados nessas disposições."

Já o artigo 22 aborda especificamente a questão das "decisões individuais automatizadas, incluindo definição de perfis" e dispõe, no 22 (1), que "o titular dos dados tem o direito de não ficar sujeito a nenhuma decisão tomada exclusivamente com base no tratamento automatizado, incluindo a definição de perfis, que produza efeitos na sua esfera jurídica ou que o afete significativamente de forma similar".

De acordo com o artigo 22 (2), esta previsão do 22 (1), não se aplica nos seguintes casos: 
a) For necessária para a celebração ou a execução de um contrato entre o titular dos dados e um responsável pelo tratamento; b) For autorizada pelo direito da União ou do Estado-Membro a que o responsável pelo tratamento estiver sujeito, e na qual

estejam igualmente previstas medidas adequadas para salvaguardar os direitos e liberdades e os legítimos interesses do titular dos dados; ou c) For baseada no consentimento explícito do titular dos dados.

Conforme o artigo 22(3), nas hipóteses das previsões do $22(2)^{13}$ (a) e (c) serão aplicadas pelo responsável pelo tratamento de dados, medidas para salvaguardar os direitos e legítimos interesses do titular de dados, [...] designadamente o direito de, pelo menos, obter intervenção humana por parte do responsável, manifestar o seu ponto de vista e contestar a decisão".

Diante desta breve apresentação, que não pretende esgotar o tema proposto, é possível conhecer os dispositivos mais relevantes do Regulamento Geral de Proteção de Dados Pessoais da União Européia para as discussões e debates acerca das decisões automatizadas e seu reflexo para o reconhecimento do direito que vem sendo denominado de "direito à explicação", que será analisado no próximo tópico.

\section{REGULAMENTO GERAL DE PROTEÇÃO DE DADOS DA UNIÃO EUROPÉIA E A PREVISÃO DO DIREITO À EXPLICAÇÃO DAS DECISÕES AUTOMATIZADAS}

A partir da previsão de alguns dispositivos do próprio Regulamento Geral de Proteção de Dados Pessoais da União Européia-GDPR ${ }^{14}$ que foram apresentados no tópico anterior - passa-se a mencionar que a normativa exige o direito de explicação dos modelos de aprendizado de máquina (BURT).

Considera-se que as decisões automatizadas, que prescindem de qualquer intervenção humana "[...] parecem ir contra a noção de autonomia e personalidade no Regulamento Europeu." De modo que "[...] a orientação do Regulamento mensurar o direito à explicação busca 
fornecer alguma informação significativa sobre como os dados pessoais são utilizados em decisões automatizadas"(POLIDO et al [s/d], p.11,12).

Sob esta perspectiva fala-se, então, sobre uma nova categoria de direito a partir do GDPR, relacionada com a oposição à tomada de decisão automatizada, fazendo-se referência aos artigos “22 (decisões individuais automatizadas, incluindo definição de perfis) e sua conexão com os arts. 13 a 15 ('Informação e acesso aos dados pessoais') do Regulamento (POLIDO et al [s/d], p.12). Para além desses artigos, BURT cita ainda, o artigo 21, destacando que tanto este quanto o artigo 22 se enquadram na Seção 4 (lidando especificamente com o direito do sujeito à objeção da tomada de decisão automatizada) (BURT).

Assim, tal direito derivaria de três bases jurídicas possíveis encontradas no Regulamento:

[...] salvaguardas contra o processo automático de tomada de decisões, conforme exigido pelo artigo $22 .{ }^{\circ}$, n.. 3 , e comentado pelo considerando 71; deveres de notificação previstos nos artigos 13.. a 14..$^{\circ}$, comentados nos considerandos 60 a 62; ou o direito de acesso nos termos do artigo

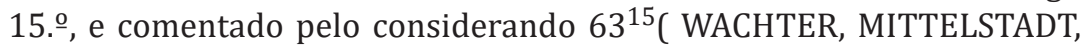
FLORIDI, 2017, p.4).

Há considerações no sentido de que o direito à explicação das decisões automatizadas consiste num tipo de retomada e atualização de previsões da Diretiva de Proteção de Dados da União Européia (DPD) de 1995.

Nesse sentido, de forma superficial e sem considerar as diferenças críticas entre as duas legislações, Goodman e Flaxman (2017, p.2) mencionam que o Regulamento vem reafirmar o direito da DPD à explicação e restrições na tomada de decisões automatizadas). Em sentido similar, há afirmações de que o regulamento atualiza os direitos da DPD sobre a tomada de decisões automatizadas sendo esta atualização um marco para o que se passou a denominar de direito à explicação (VOGL, FARHANGI, CASEY, 2018).

Independentemente de sua origem, a realidade é que a partir do Regulamento Geral de Proteção de Dados da União Européia surgiram diversos e variados debates acerca da existência, abrangência ou mesmo inexistência do chamado direito à explicação das decisões automatizadas. 
Enquanto alguns estudiosos posicionam-se "veementemente contra a mera possibilidade de tal direito existir", outros, como o Escritório do Comissário de Informações do Reino Unido, parecem achar que o direito é claramente evidente ${ }^{16 " \text { (BURT). }}$

Há afirmações de pesquisadores, órgãos governamentais e da mídia no sentido de que o "direito à explicação" de decisões tomadas por sistemas algorítmicos automatizados e artificialmente inteligentes é legalmente exigido pelo GDPR assim como há pesquisadores, que foram pioneiros nesse debate, que se posicionam no sentido de que "existem várias razões para duvidar da existência, escopo e viabilidade de um "direito à explicação" de decisões automatizadas”(WACHTER, MITTELSTADT, FLORIDI, 2017, p.1,2).

Os debates envolvem, dentre outros, questionamentos no sentido de saber se a transparência exigida deve estar relacionada ao compartilhamento do funcionamento do algoritmo, que é tido como uma 'caixa preta', com os sujeitos de dados afetados pelo algoritmo ou se, em razão de esta informação não ser de fácil compreensão, seria necessário o fornecimento de uma explicação sobre esse funcionamento (SCIENCE AND TECHNOLOGY COMMITTE,2018, p.30).

As controvérsias levantam, também, questões relativas às possibilidades de aplicação dessa nova categoria de direito e sobre como ela será colocada em prática de forma efetiva (POLIDO et al, [s/d], p. 12).

Pesquisadores da Oxford Internet Institute publicaram um artigo sob o título Por que um direito à explicação da tomada de decisão automatizada não existe no Regulamento Geral de Proteção de Dados no qual argumentam que o Regulamento não implementa o direito à explicação mas sim o que eles denominam de direito limitado a ser informado ${ }^{17}$. Para tanto, fazem uma análise com intuito de refutar as três bases jurídicas citadas acima- que possibilitariam a existência do direito à explicação da tomada de decisão automatizada no GDPR ${ }^{18}$ (WACHTER, MITTELSTADT, FLORIDI, 2017, p.2; 4-10).

Em seu trabalho, eles elaboraram dois tipos de elucidações ${ }^{19}$ relacionadas ao que se entende por esse direito à explicação a partir do GDPR (WACHTER, MITTELSTADT, FLORIDI, 2017, p.3,4), estabelecendo as diferenças entre: 1) explicações relativas à funcionalidade do sistema, ou 
seja, a lógica, significado, conseqüências previstas e funcionalidade geral de um sistema de tomada de decisões automatizado, [...]" e explicações das decisões específicas, ou seja, a lógica, razões e circunstâncias individuais de uma decisão automatizada específica,[...]" ; 2) explicação ex ante, que ocorre antes de uma tomada de decisão automatizada, que poderia abordar apenas a funcionalidade do sistema e explicação ex post ,dada após uma decisão automatizada ter ocorrido, sendo que esta poderia abarcar tanto a funcionalidade do sistema quanto a lógica de uma decisão específica.

Essa diferenciação tem, ainda, reflexos em diferentes pesquisas sobre o tema e ela é fundamental para compreender que os próprios autores que a elaboraram negam a existência de um direito de explicação que seja ex post e de decisão específica (CANUT, MEDEIROS, 2020, p. 428).

Dentre aqueles que reconhecem o direito à explicação a partir do GDPR estão Goodman e Flaxman. Eles fazem referência - mas sem elaborar uma análise mais profunda - aos artigos 13-15 e ao 22 do Regulamento, e mencionam os possíveis desafios a serem enfrentados pelos engenheiros na adaptação a essa normativa, destacando que tal adequação imporá requisitos ao design algorítmico e exigirá mais investigação (2017,p.3; 6,7).

Apesar de reconhecerem a desavença acerca da existência do "direito de explicação" no GDPR, Selbst e Powles ${ }^{20}$ (2017, p.233; 235) elaboraram uma pesquisa com o intuito de "reorientar esse debate mostrando que o texto puro do GDPR apóia esse direito", o que seria visível diante de uma leitura clara dos Artigos 13 (2) (f), 14 (2) (g), 15 (1) (h) e 22. Para eles, o debate sobre o "direito à explicação" envolve questões complicadas, complexas e de um alto desafio técnico relacionadas aos sistemas de aprendizado de máquina e de inteligência Artificial.

Após análise detalhada e crítica do trabalho de Goodman e Flaxman, considerando-o limitado por reconhecerem um direito de explicações específicas sem fundamentos suficientes, e daquele desenvolvido pelos pesquisadores da Oxford Internet Institute ,-WACHTER, MITTELSTADT, FLORIDI - os autores seguiram no sentido de que o direito de explicação abrange decisões específicas (SELBST, POWLES, 2017, p. 253;238) e concluíram que 
Os artigos 13 a 15 fornecem direitos a'informações significativas sobre a lógica envolvida' em decisões automatizadas. Achamos que faz sentido chamar isso de direito à explicação, mas esse ponto é menos importante do que a substância do direito em si. Acreditamos que o direito à explicação deve ser interpretado funcionalmente, com flexibilidade e deve, no mínimo, permitir que um titular de dados exerça seus direitos de acordo com o GDPR e a lei de direitos humanos ${ }^{21}$ (SELBST; POWLES, 2017, p.241).

Vogal, Farhangi e Casey, chegam a mencionar que o regulamento fornece um "inequívoco 'direito à explicação' com amplas implicações legais para o design, prototipagem, testes de campo e implantação de sistemas automatizados de processamento de dados ${ }^{22 "}$ (VOGL, FARHANGI, CASEY, 2018).

Há, ainda, quem considere que o Regulamento não garante um direito integral à explicação. Essa interpretação ressalta que as disposições do Considerando 71, que se refere a explicação de uma decisão automatizada individual em si, consiste em uma disposição não vinculativa e que assim o é por opção do próprio regulamento que, diferentemente, garantiu caráter vinculativo às previsões sobre decisões automatizadas dos artigos 13 a 15 ao incluí-las nos dispositivos do GDPR sendo tais dispositivos, diferentemente do considerando 71 , relacionados ao direito de obter informações significativas sobre a lógica subjacente e o significado dos sistemas de aprendizagem de máquinas e não às decisões automatizadas individuais (BURT).

Destacando que a explicação exata de como uma decisão individual automatizada (prevista no considerando 71) foi tomada é extremamente difícil em sistemas de aprendizado de máquinas, BURT considera que essa previsão consiste em uma sugestão dos redatores do Regulamento mas não uma determinação. E, por isso, para ele, a relação do direito à explicação a uma decisão específica "se tornou um assunto polêmico"(BURT).

Por outro lado, há entendimentos de que o direito à explicação das decisões automatizadas simplesmente não existe. Em uma postagem sob o título Right to Explanation: a Right that Never Was (in GDPR), afirma-se que "a crença de que o GDPR incorpora o Direito à Explicação é uma combinação de notícias antigas, pensamento positivo e clicagem 
na internet, sendo que o GDPR, em seus 11 capítulos não menciona tal direito especificamente em nenhum deles ${ }^{23}[\ldots .$.$] "(EVE, 2018).$

Nesse sentido,

[...] Sabemos que estava sendo discutido: a regra chegou a algumas versões públicas, mas foi retirada da versão final da legislação. A essência dessas conversas ainda é vívida na coletânea de artigos do Capítulo 3, também conhecida como Direito à Informação. Concede ao titular dos dados o direito de conhecer "as finalidades do processamento para o qual os dados pessoais são destinados (...), a existência de tomadas de decisão automatizadas, incluindo a criação de perfis, (...) e, pelo menos nesses casos, informações significativas. sobre a lógica envolvida : Como apontado no relatório da Universidade de Oxford, há uma certa disparidade entre lógica e significado. 0 Artigo 22 apela à automatização afirmando que "o titular dos dados tem o direito de não estar sujeito a uma decisão baseada apenas no processamento automatizado, incluindo a criação de perfis, que produza efeitos legais sobre ele ou afeta significativamente a pessoa'. No entanto, o que temos aqui é o direito de optar pelo processamento automático: não o direito de receber um tutorial personalizado do YouTube sobre a tomada de decisões no computador ${ }^{24}$ (EVE, 2018).

Dos breves pontos controvertidos apresentados, observa-se que as discussões não se limitam a afirmações acerca da existência ou não existência do direito à explicação das decisões automatizadas. Elas se estendem à própria compreensão desse direito, no sentido de saber se ele abrange a explicação apenas da lógica do sistema ou se ele também se refere à explicação das decisões concretas individualizadas (considerando 71) e, ainda, se dizem respeito a explicações anteriores, ex ante, ou posteriores, ex post, á tomada de decisão algorítmica. Há, entre as diversas análises, divergências quanto à própria interpretação e sentido dado aos dispositivos do GDPR citados no tópico anterior.

Ainda que este seja o cenário encontrado, pôde-se notar que o artigo 22, com seus incisos, é um dispositivo central dos debates sobre a o direito á explicação das decisões automatizadas, sendo o ponto chave de conexão com os artigos 13, 14 e 15.

Essa constatação tem reflexos importantes para as pesquisas sobre a temática e evidencia o cuidado a ser tomado quando da sua abordagem. Isso porque o artigo 22 carrega expressões que se sujeitam a diferentes 
interpretações como, por exemplo, no artigo 22 (1): "decisão tomada exclusivamente com base no tratamento automatizado"; "que produza efeitos na sua esfera jurídica" ou "que o afete significativamente de forma similar".

Tantos foram os debates que surgiram acerca destas e de outras questões interpretativas do GDPR e, especialmente, sobre as decisões automatizas e a formação de perfis, que o Grupo de Trabalho do Artigo 29 para Proteção de Dados, que passou a ser denominado Comitê Europeu de Proteção de Dados (MARGOT, 2019, p.194), elaborou Diretrizes/ Orientações sobre tais temas. Estas Diretrizes, que foram adotadas em 3 de outubro de 2017, com última redação e adoção em 6 de fevereiro de 2018 (GRUPO DE TRABALHO DO ARTIGO 29, 2018) incluíram várias disposições destinadas a esclarecer o "direito à explicação" (VOGL, FARHANGI, CASEY, 2018, p.171).

Importante observar que as Diretrizes não se ocupam, de forma explícita e direta, em esclarecer se existe ou não esse "direito de explicação". No entanto, abordam pontos extremamente relevantes para as discussões sobre a temática.

Um primeiro ponto a se destacar diz respeito ao reconhecimento de que o artigo 22 (1) do GDPR introduz uma proibição geral de decisões individuais exclusivamente automatizadas (p. 9;21;40) e não um direito de objeção. Isso tem reflexos teóricos e práticos fundamentais já que, sendo um proibição, ela "aplica-se independentemente de o titular dos dados adotar uma medida relativa ao tratamento dos seus dados pessoais" (p.21) e resulta na autorização de tais decisões apenas diante das exceções do artigo 22(2) (p. 25,26), citadas no tópico anterior (GRUPO DE TRABALHO DO ARTIGO 29, 2018). Observa-se que há uma limitação do campo de aplicação do "direito de explicação" já que ele só existiria no contexto de tais autorizações.

Além disso, as Diretrizes (GRUPO DE TRABALHO DO ARTIGO 29, 2018, p. 22; 23,24). restringiram o âmbito de discussões/aplicações acerca desse direito ao conceituarem decisões exclusivamente automatizadas como aquelas em que "não há nenhuma intervenção humana no processo decisório". Houve esforço para esclarecer o significado de "produção de efeitos na sua esfera jurídica" assim como o que seria "que o afete 
significativamente de forma similar", o que foi feito, em muitos casos, por meio do recurso a situações exemplificavas.

A falta de estabelecimento concreto da abrangência de tais expressões permite a abertura para interpretações diversas. 0 que pode ser percebido do exame das considerações do Comitê de Ciência e Tecnologia do Reino Unido ao mencionarem que "[...] o governo apresenta um posicionamento padrão no sentido de que nas situações em que os algoritmos afetem direitos e liberdades dos indivíduos, deverá haver explicações acerca de como tais algoritmos funcionam. Nessas ocasiões, em que o público ou seus direitos sejam afetados significativamente, acreditam "que a resposta é uma combinação de explicação e tanta transparência quanto possível" 25 (SCIENCE AND TECHNOLOGY COMMITTE, 2018, p.30,31;43).

Apesar de ainda não apresentar os contornos precisos de sua aplicação, assim como outros direitos do GDPR, o direito à explicação tem sido visto como mecanismo próspero para a promoção da responsabilização e transparência dos sistemas de aprendizado de máquinas que vem sendo cada vez mais adotados em todo o mundo. (VOGL, FARHANGI, CASEY, 2018). Por isso, devem ser observados os importantes reflexos do reconhecimento do direito à explicação das decisões automatizadas nesse modelo de sociedade e economia já que, nesse contexto, tal direito é ponto essencial para obtenção de transparência e responsabilidade dos algoritmos (SCIENCE AND TECHNOLOGY COMMITTE, 2018, p.30,31;43).

Observa-se que quadro apresentado demonstra, por um lado, que não há, ainda, um único e pacífico posicionamento acerca da existência do direito à explicação a partir do GDPR, nem do seu sentido e extensão e, por outro, coloca em evidência a relevância do debate para uma sociedade e economia baseada em dados.

\section{CONSIDERAÇÕES FINAIS}

O trabalho dedicou-se, na primeira parte, a fazer uma breve introdução a respeito das decisões automatizadas na sociedade e economia de dados. Foi possível apresentar, de um lado, que tais decisões tornaram- 
se elemento central desses novos modelos e, de outro, que elas vêm, por vezes, gerando riscos para os direitos daqueles que estão sujeitos a elas.

Num trabalho jurídico sobre tema tão recente, relacionado com o desenvolvimento das TICs, envolvendo variáveis como algoritmos e IA, esse tópico foi fundamental tanto para justificar e evidenciar a relevância do objeto de estudo do artigo quanto para ressaltar a urgência de debates mais detidos acerca de um "direito de explicação" das decisões automatizadas.

No segundo tópico, após rápida apresentação do intuito e âmbito de aplicação do GDPR, ocupou-se em expor os dispositivos -vinculativos e não vinculativos- mais relevantes sobre decisões automatizadas. Consideraram-se relevantes aqueles dispositivos mais mencionados nos debates apresentados nas pesquisas relatadas no tópico três do trabalho.

Restou claro que a proteção do GDPR abrange tratamentos de dados pessoais total ou parcialmente automatizados, tendo sido destacados, também, alguns princípios essenciais conectados a tais decisões como o da transparência e do tratamento equitativo. Dois Considerandos tiveram destaque: o 63 e o 71 .

Dos dispositivos vinculativos foram destaque os artigos 13 (1) e (2), 14 (1) e 15 (1), que dizem respeito a direitos dos titulares de dados de obterem informações e direito de acesso. Verificou-se que a conexão desses dispositivos com as decisões automatizadas deveu-se á citação, em cada um desses artigos, ao artigo 22(1) e (4). Ao analisar o artigo 22 (1) foi possível verificar a sua limitação ás decisões tomadas exclusivamente com base no tratamento automatizado. Além disso, descreveu-se o artigo 22 (2), que apresenta exceções que excluem a aplicação do artigo 22 (1).

Foi possível perceber, também, que o GDPR não elenca um direito de explicação das decisões automatizadas no rol dos direitos dos titulares de dados, e que o termo explicação consta do Considerando 71 que, ao mencionar o direito de obter uma intervenção humana enquanto uma garantia adequada, cita o de obter uma explicação sobre a decisão tomada para que ela possa ser avaliada e contestada.

No terceiro tópico do artigo apresentaram-se posicionamentos encontrados em diversos artigos científicos, especialmente estrangeiros, sobre o direito de explicação. Constatou-se um cenário repleto de posicionamentos divergentes, alguns a favor, outros contra a existência 
desse direito, alguns ampliando o seu significado e extensão enquanto outros voltados a reduzi-los.

Além disso, identificou-se um documento fundamental para o debate ora tratado: as Diretrizes do Grupo de Trabalho do Artigo 29 sobre decisões automatizadas e formação de perfis. Embora não seja um tema pacífico, foi possível identificar a centralidade do artigo 22(1) e 22(2) para os debates , assim com o influência do Considerando 71.

Verificou-se que apesar de as Diretrizes do Grupo de Trabalho do Artigo 29 não terem se posicionado, de forma explícita, acerca da existência de um direito de explicação, nem terem se ocupado em qualificá-lo como explicação de uma decisão individual ou da lógica subjacente do sistema, nem sobre sua classificação como uma explicação ex ante ou ex post, verifica-se que um exame mais detido do documento contribui para o desenvolvimento dos debates que seguem tais classificações sem, no entanto, botar um fim a eles ${ }^{26}$.

Observou-se que alguns esclarecimentos das Diretrizes do Grupo de Trabalho 29 apresentados neste trabalho foram fundamentais para os debates do direito de explicação e, até mesmo, para refinar a delimitação do tema da presente pesquisa como, por exemplo: que o artigo 22(1) trata de uma proibição geral; que a aplicação do artigo 22 (1) restringe-se ás decisões totalmente automatizadas, e a sua não aplicação nas situações excepcionais do artigo 22 (2). Isso significa, de um lado, que o objeto do presente trabalho é o direito de explicação das decisões totalmente automatizadas e não apenas automatizadas e, de outro, que não há que se falar em direito de explicação quando a decisão totalmente automatizada se der naquelas situações do 22(2), dentre as quais se encontra o consentimento explícito, tão usualmente dado pelos titulares de dados.

A partir da análise bibliográfica, que foi posteriormente acrescida com o exame documental, esperava-se confirmar a hipótese de pesquisa de que a partir de diversos dispositivos do GDPR teria sido previsto o direito à explicação. No entanto, no curso da investigação constatou-se que não há um posicionamento pacífico acerca do tema e que os debates não se limitam ao âmbito jurídico, tendo sido verificada a intensidade das discussões, também, na área de tecnologias de informação e comunicação que, diante 
da eventual criação de tal direito, teriam que criar procedimentos e alternativas para viabilizar a sua concretização.

Apesar de a hipótese inicial não ter sido confirmada, o objetivo do trabalho, de apresentar um quadro de debates ${ }^{27}$ acerca da existência do direito à explicação a partir do GDPR, pôde ser alcançado, ainda que o conteúdo encontrado nesse quadro tenha sido diverso daquele incialmente idealizado no projeto de pesquisa. Também ficou evidente a importância da abordagem acerca do direito à explicação no contexto do desenvolvimento tecnológico atual tendo em vista as potencialidades negativas que as decisões automatizadas geram para diferentes direitos fundamentais.

Desta forma, embora tenham sido constatadas tantas divergências em torno da existência do direito à explicação a partir da GDPR, nas diversas áreas do conhecimento e a partir de interpretações e pontos de vista extremamente variáveis, ressalta-se o quão fundamental é esse debate para a atual sociedade e economia de dados que se pauta cada vez mais nas funções desempenhas por algoritmos que, por sua vez, tem alcançado altos níveis de complexidade permitindo decisões automatizadas com o uso de aprendizado de máquina e Inteligência artificial.

Espera-se que o presente estudo, de caráter inicial e exploratório do tema, possa contribuir para debates mais profundos e mais delimitados sobre o direito de explicação das decisões automatizadas.

\section{NOTAS}

1 Foi possível identificar que os artigos de Goodman e Flaxman ; Watcher et al e Selbst e Powles marcaram o início dos debates acerca da temática.

2 Opta-se por utilizar a sigla GDPR, derivada do inglês General Data Protection Regulation em razão de sua utilização nos debates internacionais.

3 Um quadro atualizado até a data de submissão do presente artigo, em novembro de 2018. Em recente publicação o tema foi retomado sob uma perspectiva comparativa com a Lei de Proteção de Dados Brasileira (CANUT, MEDEIROS, 2020).

4 Tendo em vista a recente Lei no 13.709, de agosto de 2018 - a Lei Geral de Proteção de Dados pessoais - elaborada sob influência do GDPR, a temática ora apresentada ganha maior relevo para as pesquisas e projetos nacionais.

5 Diversos autores, sob diferentes perspectivas, abordaram e, em alguns casos, ainda abordam tais transformações seja sob uma perspectiva similar a de Castells, seja sob outras perspectivas como Pierre Lévy, Jeremy Rifikin, Daniel Bell, Alvin Toffler.

6 No presente trabalho não se pretende elaborar exames acerca das diferenças entre os termos dados e informações que, muitas vezes, são utilizados como sinônimos.

7 Explicações mais detalhadas em CANUT, MEDEIROS, 2017. 
8 Tradução livre de "Whether on private social networks or in public sector courtrooms, machine learning applications are witnessing unprecedented rates of adoption due to their ability to radically improve data-driven decision-making at a cost and scale incomparable to humans. [...]"( VOGL,FARHANGI, CASEY, 2018).

9 Todas as citações podem ser conferidas nas versões portuguesa e em inglês, que foram utilizadas para a elaboração do presente tópico (JORNAL OFICIAL DA UNIÃO EUROPÉIA; OFFICIAL JOURNAL OF THE EUROPEAN UNION).

10 O regulamento explica o significado de "identificável".

11 'Profiling' means any form of automated processing of personal data consisting of the use of personal data to evaluate certain personal aspects relating to a natural person, in particular to analyse or predict aspects concerning that natural person's performance at work, economic situation, health, personal preferences, interests, reliability, behaviour, location or movements (OFFICIAL JOURNAL OF THE EUROPEAN UNION).

12 Tratamento de categorias especiais de dados pessoais 1. É proibido o tratamento de dados pessoais que revelem a origem racial ou étnica, as opiniões políticas, as convicções religiosas ou filosóficas, ou a filiação sindical, bem como o tratamento de dados genéticos, dados biométricos para identificar uma pessoa de forma inequívoca, dados relativos à saúde ou dados relativos à vida sexual ou orientação sexual de uma pessoa. 2. 0 disposto no n.o 1 não se aplica se se verificar um dos seguintes casos: ( no artigo $5^{\circ}$ )

13 De acordo com o artigo 22 (4), "as decisões a que se refere o no 2 não se baseiam nas categorias especiais de dados pessoais a que se refere o artigo 9 (1), a não ser que o no 2, alínea a) ou g), do mesmo artigo sejam aplicáveis e sejam aplicadas medidas adequadas para salvaguardar os direitos e liberdades e os legítimos interesses do titular."

14 Aqui, como explicado no tópico anterior, também será utilizada a sigla GDPR.

15 Tradução livre de:"[...] safeguards against automated decision-making as required under Article 22(3), and commented upon by Recital 71; notification duties under Articles 13-14 commented upon by Recitals 60-62; or the right of access under Article 15, and commented upon by Recital 63". ( WACHTER, MITTELSTADT, FLORIDI, 2017, p.4).

16 Tradução livre de:"Some scholars, for example, have spoken out vehemently against the mere possibility that such a right exists. Others, such as the UK's own Information Commissioner's Office, seem to think the right is pretty clearly self-evident".( BURT).

17 Sobre a existência de tomadas de decisão automatizadas e funcionalidade do sistema, conforme explicado ao longo do artigo pelos pesquisadores (WACHTER, MITTELSTADT, FLORIDI, 2017).

18 O que não será análise do presente trabalho.

19 Importante observar que tais distinções são criticadas no trabalho de SELBST e POWLES ( 2017, p.239-241)

20 Os autores tomam como base de sua análise o que eles chamaram de "contribuições mais proeminentes" sobre o debate e fazem uma crítica dos mesmos: " dois trabalhos explosivos de Oxford, que moldaram imediatamente o debate público. 80 primeiro artigo, de Bryce Goodman e Seth Flaxman, afirma que o GDPR cria um "direito à explicação", mas não elabora muito além disso. ponto. 0 segundo artigo, de Sandra Wachter, Brent Mittelstadt e Luciano Floridi, afirma que atualmente não existe tal direito(SELBST, POWLES, 2017, p.234; 237-241). Trabalhos que, por sua vez, foram referenciados no presente artigo.

21 Tradução livre de : "Articles 13-15 provide rights to 'meaningful information about the logic involved' in automated decisions. We think it makes sense to call this a right to explanation, but that point is less important than the substance of the right itself. We believe that the right to explanation should be interpreted functionally, flexibly, and should, at a minimum, enable a data subject to exercise his or her rights under the GDPR and human rights law .(SELBST; POWLES, 2017, p.241).

22 Tradução livre de: "The GDPR provides an unambiguous "right to explanation" with sweeping legal implications for the design, prototyping, field testing, and deployment of automated data processing systems." (VOGL, FARHANGI, CASEY, 2018).

23 Tradução livre de: "The belief that GDPR incorporates Right to Explanation is a combination of old news, wishful thinking, and internet clickbait. [...]" (EVE, 2018). 
24 Tradução livre de: "We know it was being discussed: the rule had made it to some public drafts, but was dropped from the final version of the legislation. The gist of these talks is still vivid in the collection of articles in Chapter 3, otherwise known as the Right to Information. It grants the data subject the right to know "the purposes of the processing for which the personal data are intended (...), the existence of automated decision-making, including profiling, (...) and, at least in those cases, meaningful information about the logic involved“. As pointed out in the Oxford University report, there is a certain disparity between logic and significance. Article 22 calls out automation by stating that "the data subject shall have the right not to be subject to a decision based solely on automated processing, including profiling, which produces legal effects concerning him or her or similarly significantly affects him or her". Yet what we get here is the right to opt-out from automatic processing: not a right to be delivered a personalised YouTube tutorial on computer decision making." (EVE, 2018).

25 Tradução livre de : [...] "Where algorithms might significantly adversely affect the public or their rights, we believe that the answers a combination of explanation and as much transparency as possible."(SCIENCE AND TECHNOLOGY COMMITTE, 2018, p.30,31;43).

26 Apesar da relevância de tais orientações e de como eles podem refletir na classificação e delimitação de um "direito de explicação das decisões automatizadas", seu exame foge ao escopo do presente trabalho.

27 Um quadro atualizado até a data de submissão do presente artigo, em novembro de 2018.

\section{REFERÊNCIAS}

ARTHUR, Lisa.What Is Big Data?Aug 15, 2013. Forbes.com. Disponível em: $<$ https://www.forbes.com/sites/lisaarthur/2013/08/15/what-is-bigdata/\#11ddbc555c85>. Acesso em jun. de 2017.

BRASIL.Ministério da Ciência, Tecnologia, Inovações e Comunicações.Câmara IoT. Consulta Pública."Identificação dos tópicos de relevância para a viabilização da Internet das Coisas no Brasil"2016. Disponível em:<http://www.abinee. org.br/informac/arquivos/aiot.pdf>. Acesso em junho de 2017.

BURRELL, jenna. How the machine 'thinks': Understanding opacity in machine learning algorithms.Big Data \& Society January-June 2016:1-12 https://doi. org/10.1177/2053951715622512.Disponível em:< http://journals.sagepub. com/doi/pdf/10.1177/2053951715622512>. Acesso em julho de 2018.

BURT, Andrew. Is there a 'right to explanation' for machine learning in the GDPR? https://iapp.org/news/a/is-there-a-right-to-explanation-for-machinelearning-in-the-gdpr/. Acesso em julho de 2018.

CASTELLS, Manuel. trad. Roneide Venâncio Majer.6 ed. A sociedade em rede. São Paulo: Paz e terra, 1999. 
CANUT, Letícia. Proteção do consumidor no comércio eletrônico. Uma questão de inteligência coletiva que ultrapassa o Direito Tradicional. Curitiba: Juruá, 2007.

CANUT, Letícia; MEDEIROS, Heloísa Gomes. Os algoritmos nas relações de consumo eletrônicas: análise do direito do consumidor à informação. GEDAI Grupo de Estudos de Direito Autoral e Industrial Prof. Dr. Marcos Wachowicz (organizador). Anais do XI CODAIP. XI Congresso de Direito de autor e interesse Público Estudos de Direito de Autor e Interesse Público. Curitiba: Universidade Federal do Paraná, 2017. Disponível em:< http://www.gedai.com.br/sites/ default/files/publicacoes/anais_xi_codaip-2017-gedai.pdf $>$.Acesso em julho de 2018.

CANUT, Letícia; MEDEIROS, Heloísa Gomes. O Direito de explicação das decisões totalmente automatizadas no RGPD e na LGPD brasileira. In: Proteção de dados pessoais em perspectiva: LGPD e RGPD na ótica do direito comparado. Organização de Marcos Wachowicz. Curitiba: Gedai, UFPR 2020. Disponível em:<https://www.gedai.com.br/wp-content/uploads/2020/11/Protecao-dedados-pessoais-em-perspectiva_ebook.pdf>.

EUROPEAN COMMISSION. Enter the Data Economy EU Policies for a Thriving Data Ecosystem. Issue 21.11 January 2017. Disponível em: <https://ec.europa. eu/epsc/sites/epsc/files/strategic_note_issue_21.pdf>. Acesso em julho de 2018.

EVE. Right to Explanation: a Right that Never Was (in GDPR). Posted on 1st March 2018 by Eve the Analyst .Disponível em:< http://datawanderings. com/2018/03/01/right-to-explanation/>. Acesso em julho de 2018.

GAL, Michal; ELKIN-KORREN, Niva. Algorithmic Consumers. (August 8, 2016). Harvard Journal of Law and Technology, Vol. 30, 2017. Disponível em < SSRN: https://ssrn.com/abstract=2876201>. Acesso em jun. 2017.

GOODMAN, Bryce; FLAXMAN, Seth. European Union regulations on algorithmic decision-making and a "right to explanation". AI Magazine, Vol 38, No 3, 2017. DOI: 10.1609/aimag.v38i3.2741. Disponível em:<https://arxiv.org/pdf/1606.08813. pdf>. Acesso em julho de 2018.

GRUPO DE TRABALHO DO ARTIGO 29.o PARA A PROTEÇÃO DE DADOS. Orientações sobre as decisões individuais automatizadas e a definição de perfis para efeitos do Regulamento (UE) 2016/679. 2018. Disponível 
em: https://www.cnpd. pt/home/rgpd/docs/wp251rev01_pt.pdf. Acesso em: maio 2020.

JORNAL OFICIAL DA UNIÃO EUROPÉIA. Regulamento (UE) 2016/679 do Parlamento Europeu e do Conselho de 27 de abril de 2016 relativo à proteção das pessoas singulares no que diz respeito ao tratamento de dados pessoais e à livre circulação desses dados e que revoga a Diretiva 95/46/CE (Regulamento Geral sobre a Proteção de Dados). Disponível em: < https://eur-lex.europa.eu/legal-content/PT/TXT/PDF/?uri=CELEX:32016R 0679\&from=PT $>$. Acesso em julho de 2018.

MARGOT E. Kaminski. The Right to Explanation, Explained. BERKELEY TECH. L.J. 189 (2019), available at https://scholar.law.colorado.edu/articles/1227. Disponí- vel em: https://scholar.law.colorado.edu/cgi/viewcontent. cgi?article=2335\&- context=articles. Acesso em: jul. 2020 .

NOVO, Rafael Fernandes; AZEVEDO, Marilia Macorin de. A individualização do consumidor por meio do avanço das redes sociais e do Big Data. Revista científica On-line de tecnologia, gestão e humanismo, v.4, n. 2 - nov. 2014. Disponível em: http://www.fatecguaratingueta.edu.br/revista/index.php/RCO-TGH/article/ view/65/74. Acesso em junho de 2017.

OECD. Organisation for Economic Co-operation and Development. Data-Driven Innovation: Big data for growth and well-being. Paris: OECD Publishing, 2015. Disponível em: http://www.keepeek.com/Digital-Asset-Management/oecd/ science-and-technology/data-driven-innovation_9789264229358-en\#page1. Acesso em junho de 2017.

OFFICIAL JOURNAL OF THE EUROPEAN UNION. Regulation (EU) of the European Parliament and of the council of 27 April 2016 on the protection of natural persons with regard to the processing of personal data and on the free movement of such data, and repealing Directive 95/46/EC (General Data Protection Regulation). Disponível em:< https://eur-lex.europa.eu/ legal-content/EN/TXT/PDF/?uri=CELEX:32016R0679\&from=PT>. Acesso em julho de 2018.

POLIDO, Fabrício B. Pasquot, et al. Instituto de Referência em Internet e Sociedade.GDPR e suas repercussões no direito brasileiro.Primeiras impressões de análise comparativa. [s/d] . Disponível em<http://irisbh.com.br/wp-content/ uploads/2018/06/GDPR-e-suas-repercuss\%C3\%B5es-no-direito-brasileiro- 
Primeiras-impress\%C3\%B5es-de-an\%C3\%A1lise-comparativa-PT.pdf>. Acesso em julho de 2018.

PUGLIESE, Márcio; Brandão, André Martins. Uma Conjectura sobre as tecnologias de Big Data na prática Jurídica. Rev. Fac. Direito UFMG. Belo Horizonte, n. 67, pp. 453 - 482, jul./dez. 2015. DOI: 10.12818/P.0304-2340.2015V67P453. Disponível em:< file:///C:/Users/usuario/Downloads/1731-3335-2-PB.pdf>. Acesso de julho de 2018.

SCIENCE AND TECHNOLOGY COMMITTE. Algorithms in decision making Fourth Report of Session 2017-19 Report, together with formal minutes relating to the report. Published on 23 May 2018 by authority of the House of Commons. Disponível em:< https://publications.parliament.uk/pa/cm201719/ cmselect/cmsctech/351/351.pdf>.

SELBST, Andrew D.; POWLES, Julia. Meaningful information and the right to explanation. International. Data Privacy Law, Volume 7, Issue 4, 1 November 2017, Pages 233-242,https://doi.org/10.1093/idpl/ipx022. p. 233- 242. Disponível em< https://academic.oup.com/idpl/article/7/4/233/4762325>. Acesso de julho de 2018.

VOGL, Roland ; FARHANGI, Ashkon; CASEY, Bryan. Rethinking Explainable Machi- nes: The Next Chapter in the GDPR's 'Right to Explanation' Debate. 15 May 2018. Disponível em: https://www.law.ox.ac.uk/business-law-blog/ blog/2018/05/re- thinking-explainable-machines-next-chapter-gdprs-rightexplanation. Acesso em: jul. 2020.

TAURION, Cezar. Onde os algoritmos e a inteligência artificial vão nos levar? 29 de junho de 2016. Disponível em: http://computerworld.com.br/onde-osalgoritmos-e-inteligencia-artificial-vao-nos-levar. Acesso em Agosto de 2017.

TOMASEVICIUS FILHO, Eduardo. O Marco Civil da Internet e as liberdades de mercado. In: DE LUCCA, Newton; SIMÃo FILHO, Adalberto; LIMA, Cíntia Rosa Pereira de. Direito \& Internet III. Tomo II: Marco Civil da internet ( Lei n.12.965/2014). São Paulo: Quartier Latin, 2015.

VIETH, Kilian. BRONOWICKA, Joanna. Ethics of algorithms.Centre for Internet and Human Rights. Frankfurt: European University Viadrina,2015. Disponível em:<https://cihr.eu/eoa2015web/>. Acesso em: 30 set. 2017. 
VOGL, Roland; FARHANGI, Ashkon; CASEY, Bryan. Rethinking Explainable Machines: The Next Chapter in the GDPR's 'Right to Explanation' Debate. 15 May 2018. Disponível em:https://www.law.ox.ac.uk/business-law-blog/ blog/2018/05/rethinking-explainable-machines-next-chapter-gdprs-rightexplanation. Acesso em Julho de 2017.

WACHTER, Sandra; MITTELSTADT, Brent; FLORIDI, Luciano. Why a Right to Explanation of Automated Decision-Making Does Not Exist in the General Data Protection Regulation. International Data Privacy Law, Volume 7, Issue 2, 1 May 2017, Pages 76-99,https://doi.org/10.1093/idpl/ipx005. Disponível em: < https://academic.oup.com/idpl/article/7/2/76/3860948>. Acesso e julho de 2018.

Recebido em: 20-11-2018

Aprovado em: 17-12-2020

\section{Leticia Canut}

Pós-doutora (júnior) pela Universidade Federal do Paraná, bolsista no Cnpq (do Conselho Nacional de Desenvolvimento Científico e Tecnológico). Doutorado em Direito pela Universidade Federal de Santa Catarina (2013). Mestrado em Direito pela Universidade Federal de Santa Catarina (2005). Graduação em direito pela Universidade Federal de Uberlândia (2002). Professora no Centro Universitário Estácio de Sá de Santa Catarina desde 2014. Tem experiência na área de Direito, com ênfase em Direito do consumidor e novas tecnologias, direito à saúde, e direito processual civil. Advogada inscrita na OAB/SC.

E-mail: leticiacanut@gmail.com

\section{Centro Universitário Estácio de Santa Catarina}

R. Santo Antônio - Barreiros,

CEP: 88117-350 - São José - SC 